\title{
Bernard Vouilloux, Julien Gracq, La littérature habitable
}

Paris, Hermann éditeurs, 2007, 249 p.

\author{
Roland Bourneuf \\ Université Laval
}

Depuis plus d'une décennie, l'œuvre de Gracq suscite et, au sens fort, inspire des travaux brillants. Plus que d'un entraînement mimétique, il semble bien que le phénomène relève de cette force même à laquelle se réfère si souvent Gracq dans ses romans et dans ses essais et dont il se fait le canal: le magnétisme. Comme si, en effet, les exégètes en recevaient un rayonnement et qu'ils s'entendaient à le propager. Visiblement, leurs ouvrages sont portés, au-delà de l'admiration — souvent inconditionnelle - pour les mérites littéraires de l'œuvre qu'ils prennent pour objet d'étude, par une exaltation à peine retenue, 
un pétillement de l'esprit, un ébranlement profond de la sensibilité. Il n'est pas excessif de prétendre que Gracq joue ainsi pour ceux qui le suivent le rôle que Breton a joué pour lui.

Ces livres dans le sillage gracquien sont, d'une part, des essais délibérément personnels et subjectifs qui redessinent la forme d'une vie d'écrivain et en épouse la démarche créatrice, en marquant les relais, en dégageant et prolongeant le sens. Tel est l'ouvrage d'Hubert Haddad, qui porte ce titre: œuvre d'écrivain sur un écrivain. D'autre part, les universitaires pratiquent, selon leurs visées et leurs méthodes propres, une analyse plus distancée, plus traditionnelle, mais qui ne l'est que partiellement. Après Leutrat ou Guiomar, qui appartiennent à une première génération d'exégètes, sont venus entre autres Michel Murat, Patrick Marol, Jean Bellemin-Noël, Sylvie Vignes et Bernhild Boie, auteur de la très remarquable édition de la collection de la Pléiade. Bernard Vouilloux est de ceux-là.

Déjà auteur de trois ouvrages consacrés à Gracq (Gracq autographe, 1989; De la peinture au texte. L'image dans l'œuvre de Julien Gracq, 1989; Mimesis, sacrifice et carnaval dans la fiction gracquienne, 1991), Vouilloux regroupe ici quatre études précédées d'une introduction («La forme d'une œuvre»). Le titre même de l'ouvrage reprend, en l'adaptant, celui d'un poème en prose de Liberté grande: «La terre habitable» devient «la littérature habitable, ce qui marque le rapport fondamental chez Gracq, déjà perceptible dans une lecture rapide, entre le lieu et l'écriture. L'auteur le rappelle : « [...] ne nous hantent véritablement que les lieux qui réveillent et activent les tropismes imprimés par les lieux où nous nous sommes formés [...]. C'est que les lieux où nous sommes, que nous occupons ou à travers lesquels nous ne faisons que passer, 
sont à la confluence de la mémoire et de l'imagination. » (p. 7-8) Lien organique donc qui, chez l'écrivain comme chez tout être, s'identifie à la constitution d'une «bulle ». Elle est, dit Gracq, «liée à ses goûts, à sa culture, à son climat intérieur, à ses lectures et rêveries familières » qu'il promène toujours avec lui (propos recueilli par Dominique Rabourdin). Vouilloux rappelle la formule de Hölderlin, Dichterisch wohnt der Mensch ("C'est poétiquement que l'homme habite sur cette terre») et affirme que toutes les fictions de Gracq «peuvent se lire comme des rêveries sur les attaches secrètes qui relient la plante humaine à son lieu » (p. 13), l'expression figurant dans Préférences. Et de même que la terre nous nourrit et nous permet de l'habiter par ce lien organique, la littérature doit accueillir le lecteur et lui permettre de respirer : il y a non pas analogie mais identité du rapport.

Les études qui suivent cette introduction analysent de très près quelques-unes des modalités selon lesquelles Gracq, comme tout écrivain, a "bâti sa maison » en " conformité avec notre forme empreinte», forme qui est à la fois Gestalt première, dans le sens où Goethe l'entendait, modèle virtuel, et sa réalisation, sa formation, son développement : le vocabulaire ici fait problème et l'allemand qu'utilise ici Vouilloux est en effet le plus approprié.

Dans « Le lieu du sens », à partir du motif du tableau dans la crypte et de sa variante, l'intrusion dans une chambre vide, Vouilloux s'attache à un étonnant passage d'Un beau ténébreux qui peut sembler isolé et insolite dans l'œuvre graquienne: dans son «Journal », Gérard se dit touché par l'intervalle, dans l'histoire du Christ, entre la Résurrection et l'Ascension où se situe l'épisode des pèlerins d'Emmaüs. Occasion pour l'analyste 
de s'interroger sur le sacré chez Gracq et la position de celui-ci face au christianisme. Est avancée ici l'hypothèse séduisante selon laquelle le christianisme a pu « faire office d'analogon à la poétique générale dont visiblement Gracq cherche alors à poser les fondements » (p. 51).

La deuxième étude, « $\mathrm{Du}$ récit au livre», est consacrée à des distinctions génériques : récit, nouvelle, Novelle, Erzählung, roman. Le talent clarificateur de Vouilloux ne fait pas de doute, mais fallait-il revenir une fois de plus sur ce problème qui irrite et défie des générations d'universitaires et qui ressemble fort à la quadrature du cercle? Le dernier mot risque bien de ne jamais être dit et sans doute faudrait-il ici s'inspirer de Gracq lui-même, qui ne faisait pas mystère de son indifférence pour ces distinctions... Beaucoup plus neuves et stimulantes, par contre, sont les considérations sur le " régime fragmentaire », les caractères, la fonction et les possibilités du fragment dans l'œuvre gracquienne qui sera, en fin de compte, pratiqué et presque exclusivement privilégié.

Tel est l'objet de la quatrième et dernière section de l'ouvrage, qui élargit la réflexion en une "poétique et topique de la fragmentation » : elle est montrée à l'œuvre en particulier dans La forme d'une ville et Autour des sept collines, livres les moins étudiés et probablement les moins lus. Au passage, l'auteur fait justice des critiques souvent adressées à Gracq styliste, jugé « compassé, voire cérémonieux », alors que c'est là refus des facilités de l'époque et des compromissions, adéquation aux courants profonds de l'œuvre, marque d'une liberté. Liberté que Gracq montrait agissante précisément dans 
le style de Breton: modèle d'analyse dont Vouilloux s'inspire avec bonheur.

L'évidence est là : Gracq fascine, durablement, et c'est comme si l'on n'arriverait jamais à en trouver la raison. Rien, bien au contraire, n'interdit de s'exposer à ce rayonnement sombre et éclatant pour explorer les richesses de l'œuvre. C'est ce que fait Bernard Vouilloux, à la fois impliqué affectivement et intellectuellement dans son objet et détaché de lui. Alors que la critique s'est longtemps tenue à une approche impressionniste ou thématique (l'attente, le théâtre, les espaces intermédiaires, l'interaction rêve-réalité, l'appareillage, etc.) avec ce qu'elle comportait d'approximatif et de réducteur, Vouilloux, mieux outillé méthodologiquement, se consacre à ce qu'il nomme luimême "exégèse topologico-poétique ». Elle lui permet de reconsidérer des idées reçues, d'arpenter des versants moins fréquentés, de mettre à jour d'autres richesses d'une œuvre qui en regorge.

Le bénéfice pour le lecteur et l'admirateur de Gracq est inappréciable. Un risque cependant, qui tient à la fois à l'angle d'attaque - ce vocabulaire guerrier ne rendant pas compte du respect avec lequel l'exégète aborde son objet... - et à l'œuvre même, dont la densité quasiment sans faille induit aisément un commentaire tressé serré, parfois à l'extrême. Vouilloux constamment assure ses prises, avance avec prudence. Par la subtilité et l'intelligence qui se déploient ici, par le souci, qui se traduit dans l'écriture, de ne rien laisser échapper, mais par une inversion de ces qualités, l'analyse peut en certaines pages finir par faire écran. Que l'auteur voie ici moins une critique que l'aveu d'une préférence personnelle. Je souhaiterais pour ma part que le propos soit moins uniformément tendu par la 
volonté démonstrative, qu'il se permette plus souvent des libertés comme ces fragments («Départs») rédigés dans l'esprit de Lettrines. Le lecteur serait moins intimidé et il trouverait ainsi plus facilement à « se loger » dans ces analyses remarquablement conduites: elles parviendraient mieux encore à rendre la littérature « habitable». 